\title{
Characterization and Discrimination of Architectural Paint Samples
}

\author{
D.M. Wright, ${ }^{*}$ M.J. Bradley, and A.H. Mehltretter
}

Laboratory Division, Federal Bureau of Investigation, 2501 Investigation Parkway, Quantico, VA 22135-0001

The study reported here sought to update earlier work by Tippett, et al. [1]. The work described herein involved the comparative analysis and discrimination of 964 architectural paint chips.

Samples were submitted to the FBI Laboratory by forensic science colleagues in state, local, and other federal laboratories as well as from Evidence Response Team members assigned to FBI field offices throughout the country. Contributors were asked to sample architectural surfaces that contained a pigmented coating or finish that would be commonly recognized as paint. With each submission, contributors were asked to complete a questionnaire detailing basic information such as the surface color of the sample, street address of the structure sampled, the area sampled and surface conditions (e.g., wall, window frame, internal or external, direct sun exposure), the approximate age of the structure, and the date of the last paint application. Interior surfaces outnumbered exterior ones, and walls were the overwhelming substrate sampled.

Once all specimens had been collected, they were grouped by visual topcoat color into the following sets: black/gray, blue, brown/tan, green, off-white, red/pink, yellow/peach, and white. Each specimen was then macroscopically and microscopically examined such that all comparative aspects could be documented. Features of note were the topcoat color, the number and color of each layer present, and any features within a layer (e.g., air voids, relative layer thicknesses, a non-paint layer between paint layers). For all samples, the substrate type was noted during the microscopic examinations, but it was not used for inter-comparison purposes. Upon completion of the microscopical examinations, 20 specimens were removed from the sample set (e.g. lost, not paint, or suspected asbestos). Of the remaining 964 paint samples, the largest group was the off-white set which consisted of 298 samples. Next was the white set, which contained 197 samples, followed by green with 98. Figure 1 depicts the number of specimens in each color category.

Pair-wise comparisons of samples within each color set were then conducted. Only specimens with the same paint layer sequences (number and color of the layers) were intercompared. As needed, some specimens were compared in more than one color group in order to ensure all appropriate comparisons were conducted. Microscopical comparisons of a majority of the hued samples were straightforward and proved to be the most discriminating technique. FTIR analysis of the topcoat layer further served to greatly reduce the number of pairs requiring additional analysis. However as Figure 1 demonstrates, about half of the submitted specimens were off-white or white, necessitating a different approach for these specimens. Initial inter-comparisons of the white group, as well as those off-white specimens requiring comparison to the white paints, were accomplished via FTIR analysis. Once this process yielded smaller subsets of chemically consistent samples, a combination of surface color, surface sheen, and layer structure was used to further compare specimens within each discrete grouping.

The use of macroscopic and microscopic evaluations in concert with FTIR resulted in an overall discrimination of 99.991\%. Adding SEM/EDS and Py-GC/MS to the analytical suite resulted in 25 
additional pairs being discriminated. At the conclusion of the study, 11 pairs of samples remained undifferentiated. Figure 2 depicts an example of one of the indistinguishable pairs. However, each sample within a pair was confirmed to have been collected from the same building or structure based on evaluation of the paper work submitted with each sample. Therefore, no random pairs were left undiscriminated in this study.

An architectural paint case will also be presented to demonstrate how these findings can be used to assess the probative value of an architectural paint comparison in which the submitted samples are concluded to be associated.

\section{References}

[1] Tippett, et al. J. For. Sci. Soc. 8 (1968) 61.

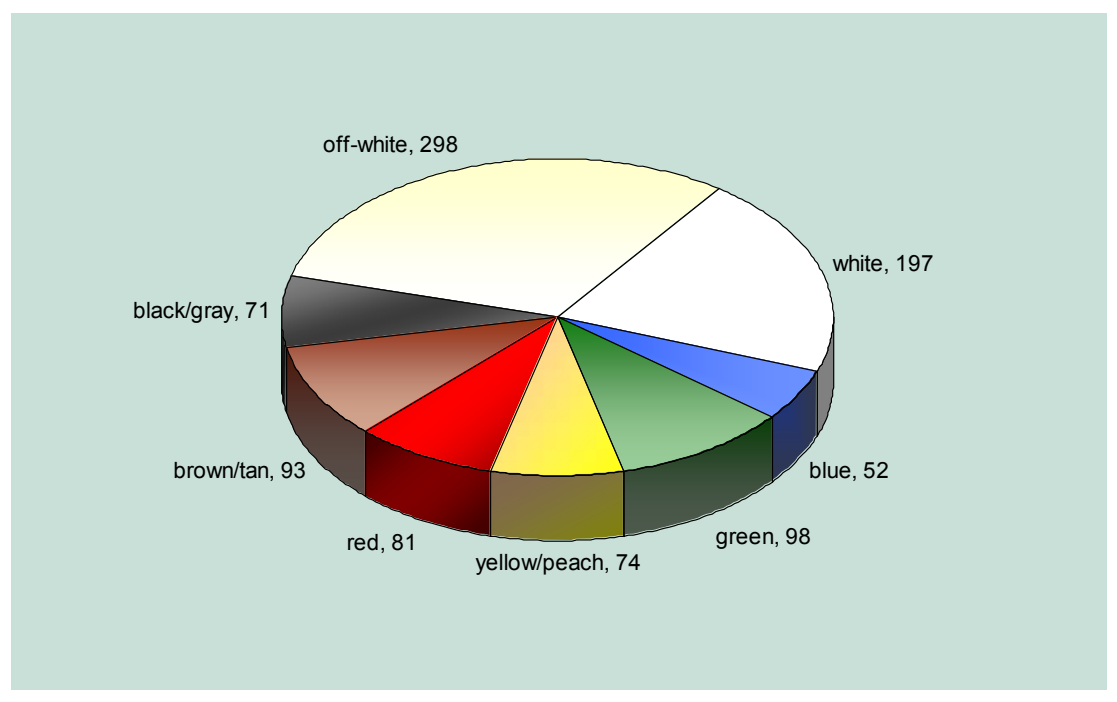

FIG.1. Breakdown of submitted architectural paint samples by color.
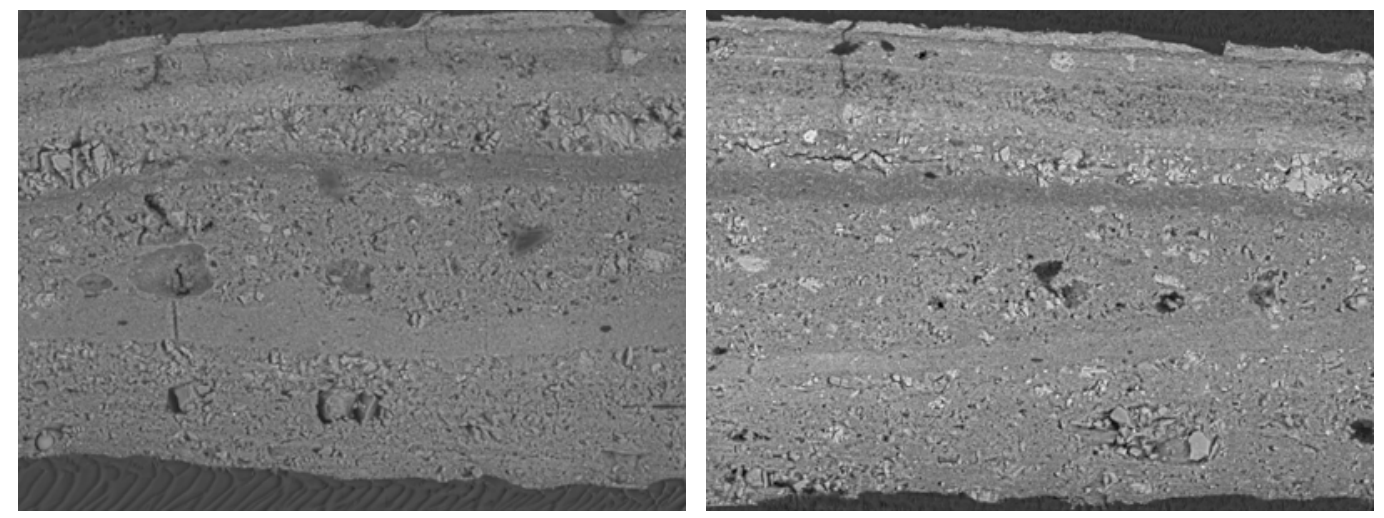

FIG. 2. Comparison of backscatter electron images of two 10-layer paint samples. 